\title{
OPEN SYSTEM FOR ROAD INFORMATION SUPPORT
}

\author{
Agata Ligier $^{1 *}$, Johannes Fliedner ${ }^{1}$, Juha Kajanen ${ }^{2}$, François Peyret ${ }^{3}$ \\ ${ }^{1}$ Institute of Construction Management and Machinery (IMB), University of Karlsruhe, \\ Am Fasanengarten, 76128 Karlsruhe, Germany, tel. +49 7216083884 , \\ e-mail: ligier@imb.uni-karlsruhe.de, fliedner@imb.uni-karlsruhe.de \\ ${ }^{2}$ Tekla Oyj, Koronakatu 1, ESPOO 02210, Finland, tel. +35898879544 \\ e-mail:juha.kajanen@tekla.com \\ ${ }^{3}$ Laboratoire Central des Ponts et Chaussées, BP 4129, 44341, Bouguenais Cedex, France, \\ tel.+33240845940,e-mail: francois.peyret@lcpc.fr
}

\begin{abstract}
Open System for Road Information Support is an open, supportive system for construction, maintenance and rehabilitation of a road, mainly focused, but not limited to the flexible pavement (asphalt). The project aims towards an European standard for a work documentation and work support system for a road worksite.
\end{abstract}

Keywords: road construction, site information systems, digital work documentation, road product model, quality management, XML, CAN, Web

\section{INTRODUCTION}

\subsection{Background and goals of the project}

The physical tools and the human know-how which are necessary to build good quality infrastructures are now at the right level. More and more equipment is powerful, techniques and tools are accurate, people are skilful. However, still much time and money are lost all along the various phases of construction, during these phases and in between, due to the lack of information and the very poor quality of the management of this information, making very small use of the new available IT.

The new contract models, i.e. "Public-PrivatePartnership" (PPP) or "Build-Own-OperateTransfer" (BOOT) and functional contracts put more responsibility on the contractor side, encouraging him to develop his own methodology and knowledge-base. There emerge a need for a quality assurance system, which would provide both the contractor and his customer with a continuous job documentation.

Clearly, the new challenge in the construction world is now the information management.

There exists on the market a range of separate digital systems to support certain phases of the road construction: design, setting-out, levelling, compaction and assessment. However, the results of any of the construction phases cannot be used as an input for the next one given the non-compatibility of the different systems. Moreover, the mentioned systems are generally not component-oriented and not configurable (except for the levelling sensors).
More and more, the construction machines are equipped with sophisticated sensors and digital networks, but the digital data are lost after the work due to lack of mobile services and database infrastructure.

The OSYRIS project aims at filling these missing links and providing a common infrastructure with open interfaces, of which the specialised components may take the advantage.

OSYRIS is an open, supportive system for construction, maintenance and rehabilitation of roads, mainly focused, but not limited, to the flexible pavement (asphalt). The project aims towards an European standard for a documentation and support system for a road worksite.

At the end of the project a line of compliant preindustrial product prototypes focused on asphalt paving and compaction operations will be presented in a field test and progressively exploited. The open set of protocols and common services will be published.

The OSYRIS project was started in February 2000 for the next three years by five partners: University of Karlsruhe (IMB) - Germany, LCPC France, Moba - Germany, Tekla Corp. - Finland, Skanska - Sweden, under the European Union Program "Growth" (Contract No: G1RD-CT199900080).

\subsection{Main features}

The OSYRIS system philosophy lies in openness and modularity The system is based on compliant off-machine components. 
Scalability and modularity of the system offers the user a real opportunity to select from the system only those parts that are really needed and run the system as small or large as the user wants. No useless functionality is necessary to purchase. Openness and modularity provide the possibility to incorporate the third party components, and to develop the system later in the direction of the user needs. The system can be easily updated when significant developments emerge, or can be expanded to additional machines for example graders, bulldozers, milling machines etc.

OSYRIS data storage and management is based upon a product model of the road, especially designed in order to be compatible with the latest road management databases, object oriented, and geographically located in $3 \mathrm{D}$. This way, the documentation files coming from the work sites using OSYRIS, would be immediately used by the new road data management systems under development at the national road authorities.

\subsection{Expected benefits}

The benefits of the use of OSYRIS system will be double: on the short term and on the long term.

On the short term, direct savings for contractors will mainly come from the use of smart control and documentation systems running permanently on the various pieces of equipment (pavers, rollers, etc.). The system can significantly reduce the number of traditional punctual tests and provide the contractor with a digital, continuous quality management system. Both road-owners and contractors will also save significant time and reduce the risks of error when using OSYRIS files for their daily exchanges.

On the long term, road owners will make benefit from using the information provided by the OSYRIS documentation by saving additional surveys, always costly and dangerous, and keeping relevant information for the next phases of road life cycle, as maintenance or rehabilitation works.

\section{SYSTEM ARCHITECTURE}

\subsection{Components}

The component based solution offers scalability and extendibility for OSYRIS system. It is as easily used in small work sites as in large-scale constructions. Therefore, the system has to manage consecutively very different amounts of data and there should not be significant differences in its performance. System's internal design influences much to the scalability of the system. Hence, a modular, component based design is more preferred, although it makes the system design much more demanding.

OSYRIS system is built on three main component levels: office, on-board computer and measurement system. The components are assigned to one of the levels, only the framework components, i.e. Product Model and Mobile Services reside on all levels, linking them together (Fig. 1). The OSYRIS framework alone does not provide any end-user functions. The functionality of the system results from the functions provided by the components.

In the run of the project the components mentioned on the Fig. 1 will be developed. 
datagrams is used for online exchange of process information between the machines

- high level, file-oriented interface based on exchange of XML files is used to exchange bulky geometry and achieved work data

- remote file system access, configuration etc. at an OS level via network removable drive

On the low level there is a continuous data transfer to guaranty the nearly real time communication. Moreover, the services are supporting also the request-response schema. Any information can be asked by any component from any other component at any time.

\subsection{Interfaces}

Following the open philosophy of the OSYRIS system, the component interfaces will be public and accessible to any party interesting in connecting to the framework on any of the levels.

There are two levels of interfaces. The first one specifies exchange rules between the components. Any third party component, e.g. an asphalt plant acquisition module, can take the advantage of it and deliver data to the common data storage.

The second level is internal to a single component and allows to extend its functionality by supplying for example an additional algorithm.

It is foreseen to built the interfaces for the specialised road owner databases when such formats will be specified.

\subsection{Web technology}

New Internet based solutions, WWW, and ebusiness offer some interesting challenges. They have caused changes in the work environment and created new needs for information distribution and analyses. As well, the amount of information transfer inside organizations and to the external customers has increased and it needs methods that are easier and more efficient to use.

In the OSYRIS system, well-proven server-client technology is used. It includes efficient data transfer in different data formats. Data transfer and delivery is controlled by the system User access management and authentication are as well supported by the server application.

\section{MEASUREMENT AND CONTROL SYSTEMS}

\subsection{Architecture}

In the OSYRIS project it is also intended to develop and demonstrate new types of on-machine, real-time measurement systems, providing key information for an efficient control of the machines and rich documentation system.

The design of Measurement and Control Systems must be based on modern communication network.
The Controller Area Network CAN exhibits a highly reliable and predictable behaviour, as it is required by embedded real-time control applications.

A further approach for the on-machine communication structure is to use standardised protocols, which gives the different electronic modules the opportunity to communicate on the same rules.

Therefore the measurement systems are based on CANopen network protocol standard. CANopen ensures easy expanding of the on-machine network and provides possibility to merge a wide range of sensors from many manufacturers, as well as data coming from the machine.

\subsection{Paver Measurement and Control System (PMCS)}

The PMCS is based on the standard levelling control for the screed. Furthermore it allows to plug in the following newly developed sensors and systems or a subset of them:

- Thickness measurement and control

- Width measurement

- Evenness measurement and control

- Material Core Temperature measurement

- Volume measurement

- Pre-Compaction measurement

- Wind and temperature measurement

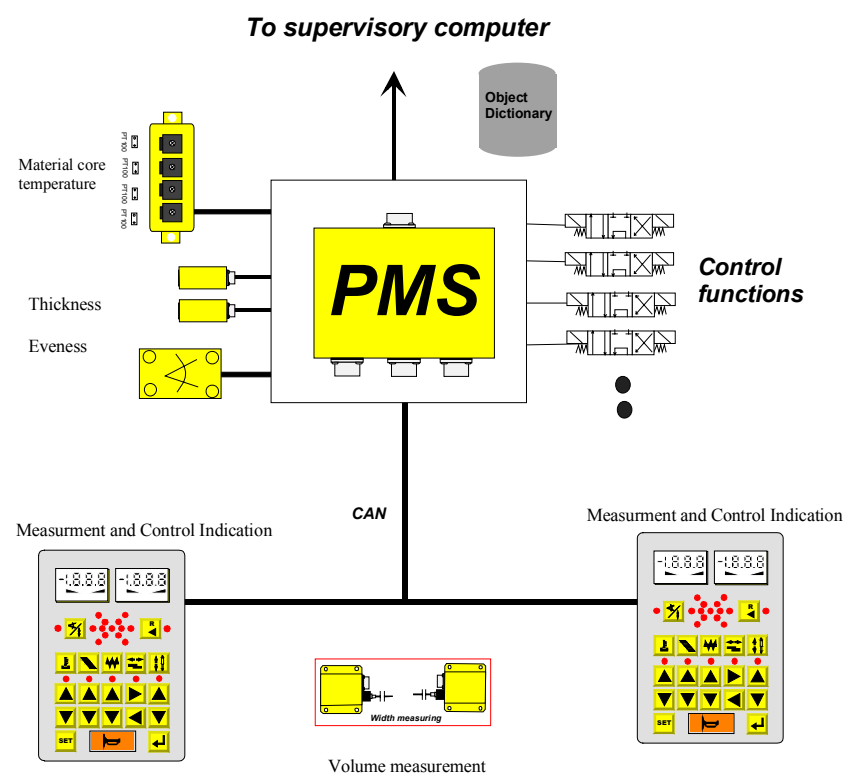

Figure 2. PMCS schema

The thickness measurement sensor is a special development for the paver, in order to measure and control the thickness of the spread material. Same concerns the width measurement of the spread layer. From those two values the material volume can be derived.

The evenness is a very important factor for laying quality and the evenness measurement can be used to better control the process of laying. 
The core temperature measurement device belongs also to the new developments for the paver. The temperature is measured on several spots inside the material close to spreading place. This measurement is a substantial part for judgement of the quality of the spread layer. The core temperature if delivered on-line to the compactor can also help to improve the compaction process.

\subsection{Pre-compaction measurement}

The degree of pre-compaction achieved by the screed of the paver is a key parameter to control carefully when laying asphalt courses. Moreover, in addition to its intrinsic importance, this parameter is fundamental for the following process, e.g. the compaction process, which will start from this point.

After a comprehensive survey of the available methods for estimating this parameter, the consortium preferred over complicated and poorly reliable methods based upon paver's parameters measurements a direct method based upon the relative measurement of the print depth of a smallscale compactor towed by the paver.

This study is currently under development and full-scale experiments are carried out this summer.

\subsection{Compactor Measurement and Control System (CMCS)}

The compaction measurement system is placed on a roller and is assessing on-line the compaction grade of the material. The first approximation is the surface coverage done with the use of GPS based positioning [1].

\subsection{Compaction degree measurement}

The compaction process requires the very right assess of the required energy. To little will mean under compaction and a drop into the life duration of the structure, to much means loss of time and money and high risks of crushing the aggregates or destroying the right profile. Unlike the soils compaction process, for which exist compaction indicators based upon the measurement of the dynamic response of the ground there are no such indicators for asphalt.

The OSYRIS study is based upon the measurement of another indicator, which is valid for any kind of material: the resisting effort to the movement of the roller due to the tenderness of the material. The most compacted is the material, the smallest is this effort.

Full-scale experimental tests have been carried out in September 2000. The post-processing and the analysis of the results confirmed that there exist a clear and reliable correlation between the estimation of this resistive effort and the material density. The estimation of the resistive effort needs very simple measurements on board (rotation angles and torque values of the 2 drums) and the dynamic model of the machine, including precise knowledge or identification of the constant parameters in it.

Another approach to the compaction assessment is based on the asphalt layer cooling model. It makes use of information like the layer thickness, core temperature at laying, surface temperature, ambient conditions and pre-compaction provided by the measurement system on the paver. This approach is possible only thanks to the OSYRIS ITinfrastructure.

\section{ON-BOARD}

\subsection{Functionality}

The on-board computer is the join between the low machine/sensor level and the office level. The main functions are:

- collect and save the measurement system data,

- display the information in a user-friendly, real world related way,

- control the machine tool in the auto mode

- prepare the achieved data for the office components,

- communicate with other parts of the working system.,

- $\quad$ self configuration.

\subsection{Features}

Following the system philosophy the on-board software supports the component based architecture.

The openness places a strong requirement on the on-board computer design. The system has to able to recognise the sources of data and its environment. One of the requirements states, that the same computer has to work on different machines, like e.g. paver and compactor. The on-board computer can work with any sensor or measurement system, which is connected to the CAN bus and supports CANopen protocol.

This feature leaves a space for third party developments and new, coming sensors. It allows to connect different data sources to the worksite information system. The encapsulation and applying of CANopen protocol allow to connect foreign data sources without a need to access the background details and implementation secrets.

The data source can be a sensor but also an algorithm, which delivers some calculated value. If required, the component can also deliver its own user interface. The third party components obtain the access to the other sensor and positioning data.

The On-board Computer software makes use of a special vectorised data handling and storage system. The so called "ribbon database" [2] is specially designed to store the process data and allow a very fast access. It reflects both: the limited storage 
capabilities on the machine and the opportunity of recovering all relevant data.

The user interface is highly configurable and can be adjusted to the current system configuration and the user needs. It is modelled on real-world and delivers the information to the operator in the friendly way.

\subsection{Positioning}

The positioning is a model example of a third party component. The system will make use of the existing solutions and adapt to the interfaces they provide. Thanks to the component architecture different positioning types can be used depending on the worksite requirements and contractor possibilities.

Beside the expensive 3D positioning (GPS, Total Station) the system supports also simple and lowcost 1D position description, based on distance measurement.

\section{OFFICE}

\subsection{OSYRIS Product Model}

OSYRIS Product Model is another part of OSYRIS system that covers the whole framework. It offers the basic management of the information through several interfaces (services) and some analysis tools for further data manipulation and utilization.

Technically, the Product Model serves as a data storage for the OSYRIS information model, and secondly it provides the information inside product model to the users thorough several interfaces. Data structure design is following the well proven patterns of relational model. Because of the aims to openness and scalability, full normalization of data is not always fulfilled. When this is allowed, the Product Model is able to support free object and property management.

Product model provides different levels of interfaces for several needs. Lowest level is programming interfaces for system's module development purposes. Well defined internal modularity and encapsulation of separate parts of the product model offers easier maintenance of the system and results in better defined functionality.

The second level of interfaces is for application development purposes. These public $\mathrm{C} / \mathrm{C}++$ interfaces offer the product model business functionality and data manipulation functionality for extended business applications and user interface development. Public programming interfaces make the product model available also for third party vendors, who may be interested to develop some functionality on top of the OSYRIS-system.

Third level of interfaces is file import/export level that provides information exchange between different systems using same data transfer formats like XML or VRML in visualization.

\subsection{Road Design application}

OSYRIS Road Design is not actually in the project specified application, but it is an example of an approach and interfacing schema to connect and improve a road design system to the OSYRIS framework. In the OSYRIS project Xroad/Xstreet Road Design system (Tekla Corp.) has been adapted and improved to offer more flexible interfacing to OSYRIS and another road design systems.

$\mathrm{Xroad} / \mathrm{Xstreet}$ is as a family of standalone products for civil engineering. It consists of applications dedicated to different parts of the design work, such as

- environment model for managing objects and attributes modelling the environment,

- road design, a powerful design tool, using creation information to make an exact 3D model of the road structure,

- $\quad$ soil investigation for managing and visualising the information acquired by common investigation methods,

- equipment application is used to create and manage both existing and planned equipment.

Each product is dedicated to one profession and can be used separately, but every product can view and query the information of all the others without file transfers or conversions. With improved interfacing, external applications like OSYRIS framework are also allowed to access all the information in the system.

\subsection{Onsite Design Viewer}

Onsite Design Viewer is an extension of the OSYRIS Road Design. It is a "light" version of the $\mathrm{Xroad} / \mathrm{Xstreet}$ system to be used on the worksite. It is designed in order to make the last road design corrections and adjustments on the worksite and to give a possibility to take into account the work already performed.

OSYRIS Onsite Design Viewer has mainly the same functionality as the $\mathrm{Xroad} / \mathrm{X}$ street, but it can be used without any separate database management system, which makes it advantageous in the worksites, where no full-scale road design system is available.

\subsection{Work Documentation}

OSYRIS Work Documentation is an application to provide the worksite and achieved work information during the construction work and offer different quality information for quality assurance purposes.

Work Documentation includes functions for displaying the information in the OSYRIS Product Model in on-screen or paper reports. Information 
can be used to make pre- or user-defined analyses for reporting the work achievements and quality. It can be also used as work control and follow-up tool.

In this way Work Documentation can deliver reports built on the parameters measured during the work, like position of the machine, speed, layer thickness on the left and the right side of the screed, core temperature, etc. But also it has an access to the information coming from other sources (at the moment typed in), like the type of material used, results of core drill tests, or machine fleet configuration.

New analyse or reporting functionality and formats can be added to the application as plug-ins.

\subsection{Worksite Web}

OSYRIS Worksite Web is a server-client based framework to deliver the information gathered in the OSYRIS Product Model. Especially Worksite Web application is planned to provide the work documentation data for the different users. This data can be presented as vector data with its properties, raster maps or as links to the written laboratory or work reports.

Worksite Web application consists of the data server inside the OSYRIS framework, which offers the internal functionality like Web server, data management and user authentication. Client application that works on a web-browser can be tailored for separate user needs and restrictions.

\section{PROJECT SCHEDULE}

\subsection{Requirement Specification and Design Phase}

The OSYRIS-project was started in February 2000 and shall be closed in the beginning of 2003.

During the first year OSYRIS mainly dealt with defining the requirements and designing the system and its components. In the requirements specification process the various end user groups were involved. The End Users' Club was brought to live at the beginning of the project. It involves machine manufacturers, road and earthwork contractors, road owners, standardisation and lobbying organisations.

Several feasibility studies have been carried out during the first half of the project in parallel to the mastering of the requirement specification. The studies covered the development fields of the highest risk.

In the design the emphasis was put on the openness and component architecture, meaning, that the system shall be open to user defined combination of the components and assure the possibility to link to new evolving technologies like new measurement systems or quality assurance specifications. The design is based on the most advanced and spread technologies for information storage and exchange.

\subsection{Development and Integration Phase}

At the half of the project, in August 2001 the ready components will be integrated and tested on a test track. The components do not support the full functionality yet. The foreseen configuration consist of

- the paver equipped with

- the PMCS with thickness, width, evenness, core temperature, volume measurement and control,

- the On-board Computer capable of storing and displaying the data, configurable on fly,

- wireless communication module,

- the office computer with

- the Road Design and Onsite Design Viewer with environmental data, attribute modelling and road structural modelling,

- the Work Documentation capable of displaying the achieved work and a fleet configuration.

- basic Worksite Web infrastructure to presenting work documentation data in WWW.

After the tests the demonstrations to different user groups will be given and their feed back will be taken into account in the further developments.

\subsection{Worksite validation and pre-industrialisation}

During 2002, the final year the project, the further developed components will be tested on Skanska construction sites. An intensive work site validation will be carried out during this time. Results of the worksite trials will be used for turning the prototypes into validated products.

This phase will be accompanied by worksite demonstrations to promote OSYRIS framework and to advertise the components among various user groups.

\section{Acknowledgements}

The project is funded by the European Union within the fifth framework within the thematic "Growth"-program (Contract No: G1RD-CT199900080).

\section{REFERENCES}

[1] LH. Pampagnin, F. Martinez, F. Peyret and al., "A new GPS-based guiding system for compactors", Proc. 15th International Symposium on Automation and Robotics for Construction, Munich, pp. 153162,1998

[2] J. Jurasz "Universal Digital Environment Model for Computer Integrated Road Construction", to appear in Proc. 18th International Symposium on Automation and Robotics for Construction, Krakow, 2001 